\title{
Spirulina supplementation in a mouse model of diet-induced liver fibrosis reduced the pro-inflammatory response of splenocytes
}

\author{
Tho X. Pham ${ }^{1}$, Yoojin Lee ${ }^{1}$, Minkyung Bae ${ }^{1}$, Siqi Hu${ }^{1}$, Hyunju Kang ${ }^{1}$, Mi-Bo Kim ${ }^{1}$, Young-Ki Park ${ }^{1}$ and \\ Ji-Young Lee ${ }^{1,2 *}$ \\ ${ }^{1}$ Department of Nutritional Sciences, University of Connecticut, Storrs, CT 06269, USA \\ ${ }^{2}$ Department of Food and Nutrition, Kyung Hee University, Seoul, 02447, South Korea
}

(Submitted 18 October 2018 - Final revision received 4 January 2019 - Accepted 11 January 2019 - First published online 26 February 2019)

\section{Abstract}

Treatment of liver fibrosis is very limited as there is currently no effective anti-fibrotic therapy. Spirulina platensis (SP) is a blue-green alga that is widely supplemented in healthy foods. The objective of this study was to determine whether SP supplementation can prevent obesity-induced liver fibrosis in vivo. Male C57BL/6J mice were randomly assigned to a low-fat or a high-fat (HF)/high-sucrose/high-cholesterol diet or an HF diet supplemented with $2.5 \% \mathrm{SP}(\mathrm{w} / \mathrm{w})(\mathrm{HF} / \mathrm{SP})$ for 16 or 20 weeks. There were no significant differences in body weight, activity, energy expenditure, serum lipids or glucose tolerance between mice on HF and HF/SP diets. However, plasma alanine aminotransferase level was significantly reduced by SP at 16 weeks. Expression of fibrotic markers and trichrome stains showed no differences between HF and HF/SP. Splenocytes isolated from HF/SP fed mice had lower inflammatory gene expression and cytokine secretion compared with splenocytes from HFfed mice. SP supplementation did not attenuate HF-induced liver fibrosis. However, the expression and secretion of inflammatory genes in splenocytes were significantly reduced by SP supplementation, demonstrating the anti-inflammatory effects of SP in vivo. Although SP did not show appreciable effect on the prevention of liver fibrosis in this mouse model, it may be beneficial for other inflammatory conditions.

Key words: Liver fibrosis: Spirulina platensis: Blue-green algae: Inflammation: Splenocytes

The incidence of non-alcoholic fatty liver disease (NAFLD) has increased with a rise in the obesity epidemic, with an estimated prevalence of $24 \%$ worldwide ${ }^{(1)}$. In 2016, the annual direct medical cost of NAFLD is estimated to be $\$ 103$ billion for the USA, while the total estimated cost for Germany, France, Italy and the UK is $€ 35$ billion $^{(2)}$. NAFLD is a spectrum disease that encompasses simple steatosis, steatohepatitis, fibrosis and ultimately cirrhosis $^{(3)}$. The 'two-hit model' for NAFLD development and progression includes simple steatosis with fat accumulation in the liver, that is, the first hit, followed by the second hit for the progression of steatosis to steatohepatitis ${ }^{(4)}$. The second hits include oxidative stress, lipid peroxidation, pro-inflammatory cytokines, adipokines and mitochondrial dysfunction ${ }^{(4)}$. Insulin resistance also plays a prominent role in the progression of NAFLD, as $70-80 \%$ of obese and diabetic patients have NAFLD ${ }^{(5,6)}$.

When liver injury occurs, macrophages secrete cytokines such as transforming growth factor $\beta 1$ (TGF- $\beta 1$ ) and TNF- $\alpha^{(7,8)}$, which can activate hepatic stellate cells (HSC) to produce extracellular matrix (ECM) as part of the wound healing process $^{(9)}$. In contrast, in NAFLD, chronic inflammation leads to a dysregulated wound healing response, resulting in excessive
ECM accumulation in the liver, that is, liver fibrosis ${ }^{(10)}$. As such, chronic inflammation drives fibrogenesis in HSC to exacerbate the progression of non-alcoholic steatohepatitis (NASH) to liver fibrosis.

The spleen is a secondary lymphoid organ that is closely associated with the liver via the portal vein system, such that splenic secretion passes through the liver before the general circulation $^{(11)}$. The precise contribution of the spleen to liver inflammation and fibrosis is not entirely clear. However, in humans, it has been demonstrated that spleen volume is increased in liver fibrosis and that the liver volume and spleen volume ratio, as measured by multi-detector computed tomography, is a good indicator of fibrosis grade ${ }^{(12-14)}$. In rodents, it has been demonstrated that the spleen is a source of inflammatory cells that migrate to the liver upon liver injury ${ }^{(15)}$. Furthermore, splenectomy in rodents has been shown to attenuate liver fibrosis, at least in part, through the attenuation of inflammatory macrophage infiltration ${ }^{(16)}$. Therefore, the spleen plays an important role in liver inflammation and fibrosis, and bioactive foods that reduce splenic inflammation may attenuate the development of liver fibrosis.

Abbreviations: ALT, alanine aminotransferase; HF, high-fat; LF, low-fat; LPS, lipopolysaccharide; NAFLD, non-alcoholic fatty liver disease; SP, Spirulina platensis.

* Corresponding author: J.-Y. Lee, fax +1 860486 3674, email ji-young.lee@uconn.edu 
To date, no single therapy has been approved for treating NAFLD, and few treatments for NASH exist ${ }^{(17)}$. Given the chronic nature of NAFLD development, lifestyle changes and dietary means appear to be important in its prevention. Spirulina platensis (SP) is a multicellular and filamentous edible blue-green alga that is found naturally in alkaline lakes. It has also been cultured in a controlled environment for human consumption and is considered a 'safe food with no side effects' by the United Nations Industrial Development Organization programme ${ }^{(18)}$. The protective effects of SP against inflammatory diseases, such as colitis, arthritis and allergic rhinitis, have been documented in several animal and human studies ${ }^{(19-21)}$, although the underlying molecular mechanisms have not been elucidated. We previously reported that IL-6 secretion from splenocytes isolated from apoE knockout mice, a model of atherosclerosis, fed SP decreased upon lipopolysaccharide (LPS) challenge and that SP supplementation reduced atherosclerotic lesion formation ${ }^{(22,23)}$. Furthermore, we also found that the organic extract of Spirulina platensis (SPE) represses the expression and secretion of pro-inflammatory cytokines with concomitant increases in histone $\mathrm{H} 3$ acetylation in macrophages ${ }^{(22)}$. Based on the findings, we sought to investigate whether SP supplementation can inhibit the development of liver fibrosis through its effect on inflammation and whole-body energy metabolism in a diet-induced mouse model of liver fibrosis.

\section{Methods}

\section{Animal care and diets}

Male C57BL/6J mice at the age of 8 weeks were purchased from Jackson Laboratories and they were housed under a $12 \mathrm{~h}$ light$12 \mathrm{~h}$ dark cycle. Mice were randomly assigned to a low-fat (LF) control ( $6 \%$ fat by weight, $n$ 15), a high-fat (HF)/high-sucrose/ high-cholesterol control (34\%/38\%/2.0\% by weight, $n 15)$ or an HF diet supplemented with SP (HF/SP; $2.5 \%$ by weight, $n 15$ ). A $2.5 \% \mathrm{SP}$ diet for mouse is equivalent to about $10 \mathrm{~g}$ of $\mathrm{SP} / \mathrm{d}$ for humans. Diet composition is listed in Table 1. SP powder (Earthrise $^{\circledast}$ Natural Spirulina) was generously provided by Earthrise Nutritionals. Mice had ad libitum access to water and food throughout the 20 weeks of the feeding period. Body weight and food consumption were recorded weekly, while plasma was collected every 4 weeks through the lateral tail vein after a $6 \mathrm{~h}$ fast. At the end of the feeding period, mice were fasted for $6 \mathrm{~h}$ and anaesthetised with ketamine $(110 \mathrm{mg} / \mathrm{kg}) / x y l a z i n e ~(10 \mathrm{mg} / \mathrm{kg})$ (Henry Schein Animal Health). Serum was collected by cardiac puncture after which the mice were euthanised by cervical dislocation before collection of liver and epididymal white adipose tissue (eWAT). All tissues were snap frozen in liquid $\mathrm{N}_{2}$ or fixed in $10 \%$ formalin. Serum and tissues were stored at $-80^{\circ} \mathrm{C}$ until analysis. All animal procedures were approved by the Institutional Animal Care and Use Committee at the University of Connecticut.

\section{Oral glucose tolerance test}

Oral glucose tolerance was assessed in the mice on an experimental diet for 18 weeks after a $6 \mathrm{~h}$ fast. Tail blood glucose was measured using an Accu-Chek Aviva glucometre at 0 , 15, 30, 60, 120 and $180 \mathrm{~min}$ after administration of $2 \mathrm{~g} / \mathrm{kg}$ body weight by oral gavage. The AUC was calculated for each mouse using GraphPad Prism 6.0 (GraphPad Software Inc.) and then averaged for each group.

\section{Indirect calorimetry}

After 19 weeks on one of the experimental diets, mice were subjected to indirect calorimetry to measure activity and energy expenditure using the Oxymax Comprehensive Lab Animal Monitoring System (CLAMS; Columbus Instruments). Mice were singly housed in each CLAMS unit for a total of $72 \mathrm{~h}$ during which they had access to their respective diet and water ad libitum. The CLAMS units were located in a temperature-controlled cabinet that was kept at $22^{\circ} \mathrm{C}$ on a $12 \mathrm{~h}$ light $-12 \mathrm{~h}$ dark cycle. The first $48 \mathrm{~h}$ served as acclimation time for the mice, and data from this period were not used for analysis. Average $\mathrm{VO}_{2}, \mathrm{VCO}_{2}$, respiratory exchange ratio, energy expenditure, $X$-axis and $Y$-axis movement were calculated by averaging all measurements in the light and dark cycle during the last $24 \mathrm{~h}$ period.

\section{Total RNA isolation and quantitative real-time PCR}

Total RNA was extracted from tissues using TRIzol reagent (Thermo Fisher) following the manufacturer's protocol. Reverse transcription for complementary DNA synthesis and quantitative real-time PCR analysis were performed as previously described $^{(24,25)}$ using a Bio-Rad CFX96 Real-Time System (BioRad). Primers were designed according to the GenBank database using the Beacon Designer 7 software (PREMIER Biosoft International). Primer sequences are available upon request.

\section{Histological analysis}

Formalin-fixed livers were sent to the Connecticut Veterinary Medical Diagnostic Laboratory (CVMDL) at the University of Connecticut for paraffin embedding and sectioning. Sections were then stained with haematoxylin and eosin (H\&E) or Gömöri trichrome by the CVMDL. Slides of stained sections were then imaged with a Zeiss Axio Vert A1 equipped with an MRc camera and $10 \times$ objectives.

\section{Splenocyte isolation}

Spleen samples were harvested from mice on experimental diets after 20 weeks at the day of killing as previously described, with minor modification ${ }^{(22)}$. Erythrocytes were removed with Histopaque 1083 (MilliporeSigma) in a $15 \mathrm{ml}$ conical tube and centrifuged at $300 \mathrm{~g}$ at $25^{\circ} \mathrm{C}$ for $30 \mathrm{~min}$ with deacceleration turned off. Splenocytes were then carefully removed from the opaque interface and washed two times with cold Roswell Park Memorial Institute (RPMI) media. Splenocytes were then plated at a concentration of $4.0 \times 10^{6}$ cells/well and treated with or without $500 \mathrm{ng} / \mathrm{ml}$ of LPS (MilliporeSigma) for $20 \mathrm{~h}$ in RPMI media.

\section{Liver lipid extractions and serum chemistries}

Liver lipids were extracted into chloroform-methanol (2:1) and solubilised in Triton X-100 as previously described ${ }^{(24)}$. Both 
Table 1. Diet composition ( $\mathrm{g} / \mathrm{kg}$ ) of a low-fat (LF) control diet or a high-fat (HF) diet containing Spirulina platensis (SP)

\begin{tabular}{lccr}
\hline & LF & HF & HF/SP \\
\hline Maize starch & $450 \cdot 0$ & $0 \cdot 0$ & $0 \cdot 0$ \\
Dextrinised maize starch & $85 \cdot 0$ & $0 \cdot 0$ & $0 \cdot 0$ \\
Sucrose & $100 \cdot 0$ & $340 \cdot 0$ & $335 \cdot 0$ \\
Casein & $200 \cdot 0$ & $200 \cdot 0$ & $184 \cdot 0$ \\
L-Cys & $3 \cdot 0$ & $3 \cdot 0$ & $3 \cdot 0$ \\
Lard & $20 \cdot 0$ & $300 \cdot 0$ & $298 \cdot 0$ \\
Soyabean oil & $40 \cdot 0$ & $40 \cdot 0$ & $40 \cdot 0$ \\
Insoluble fibre & $50 \cdot 0$ & $47 \cdot 0$ & $45 \cdot 0$ \\
AlN-93 mineral mix & $40 \cdot 0$ & $38 \cdot 0$ & $38 \cdot 0$ \\
AIN-93 vitamin mix & $10 \cdot 0$ & $10 \cdot 0$ & $10 \cdot 0$ \\
Choline bitartrate & $2 \cdot 0$ & $2 \cdot 0$ & $2 \cdot 0$ \\
Cholesterol & 0 & $20 \cdot 0$ & $20 \cdot 0$ \\
$t$-Butylhydroquinone & $0 \cdot 008$ & $0 \cdot 008$ & $0 \cdot 008$ \\
Spirulina & $0 \cdot 0$ & $0 \cdot 0$ & $25 \cdot 0$ \\
Total & 1000 & 1000 & 1000 \\
\hline
\end{tabular}

liver lipids and serum lipids were determined enzymatically using Cholesterol Reagent (Pointe Scientific) and an L-Type TG M Kit (Wako Chemicals) for total cholesterol (TC) and TAG, respectively. Serum alanine aminotransferase (ALT) activity was measured using liquid ALT (SGPT) Reagent Set (Pointe Scientific). Serum glucose was measured using Liquid Glucose (Oxidase) Reagent Set (Pointe Scientific).

\section{Statistical analysis}

The number of animals per group ( $n$ 15) was estimated using a power analysis based on our preliminary SP mouse study using apoE knockout mice to detect a $20 \%$ difference in IL- 6 secretion from splenocytes at $80 \%$ power with a two-tailed level of significance at $P=0.05(159.6($ sem 61.1) pg/ml). One-way ANOVA with Tukey's post boc test or Student's $t$ test was used to detect significant differences in treatments, with $P<0.05$ considered significant by GraphPad Prism 6 (GraphPad Software Inc.). Data are expressed as means and standard errors of the mean.

\section{Results}

\section{Effect of Spirulina platensis supplementation on liver fibrosis}

Mice fed the experimental diets progressively gained weight over the 20-week feeding period (Fig. 1(A)). While mice fed HF and $\mathrm{HF} / \mathrm{SP}$ diets gained significantly more weight than mice on the LF diet, their body weights were not significantly different from each other. At the time of killing, liver weights were significantly higher in $\mathrm{HF}$ and HF/SP groups compared with the LF group, while eWAT weights were not different between the groups (Fig. 1(B) and $(\mathrm{C})$ ).

Plasma ALT, a marker of liver damage, progressively increased in HF and HF/SP groups compared with the LF group throughout the feeding period (Fig. 2(A)). At 16 weeks, HF/SP diet-fed mice showed significantly lower plasma ALT than mice on the HF diet. The livers of mice on HF or HF/SP diets had similar levels of TC and TAG accumulation that was significantly higher than those of the LF group (Fig. 2(B)). H\&E staining of liver sections corroborated that lipid accumulation in the livers of both HF and HF/SP fed mice were similar (Fig. 2(C), upper panel). Trichrome staining, which stains collagen blue, demonstrated that HF and HF/SP diets induced noticeably more hepatic collagen accumulation compared with the LF control (Fig. 2(C), lower panel). Also, mice on $\mathrm{HF}$ and $\mathrm{HF} / \mathrm{SP}$ diets showed significantly higher mRNA expression of collagen $1 \alpha 1$ than the LF group, with no differences in $\alpha$-smooth muscle actin expression (Fig. 2(D)).

\section{Effect of Spirulina platensis supplementation on serum lipid and glucose tolerance}

Mice on HF and HF/SP diets showed significantly higher serum TC and glucose concentrations than those of the LF group; however, serum TAG levels were not different between groups (Fig. 3(A)-(C)). Mice fed HF, but not HF/SP, showed significantly impaired glucose intolerance compared with the LF control (Fig. 3(D) and (E)).

\section{Effect of Spirulina platensis supplementation on energy metabolism}

We determined metabolic rates and physical activities of mice on the experimental diets. Mice on the LF diet had a higher rate of $\mathrm{O}_{2}$ consumption and $\mathrm{CO}_{2}$ production than those of $\mathrm{HF}$ and $\mathrm{HF} / \mathrm{SP}$ groups, despite no significant differences in their activity (data not shown), indicating that their metabolic rates are higher than HF and HF/SP fed mice (Fig. 4(A)-(C)). Also, mice on HF and HF/SP diets had significantly lower respiratory exchange ratio compared with the LF group, suggesting that they favour fat over carbohydrate as energy substrates (Fig. 4(D)). Mice on the LF diet had significantly higher energy expenditure than those of HF and HF/SP groups in the dark cycle (Fig. 4(E)).

\section{Effect of Spirulina platensis supplementation on basal and lipopolysaccharide-stimulated inflammatory gene expression of splenocytes}

The spleen contributes to the development of liver fibrosis as a source of immune cell migration and splenic cytokine secretion 
(A)

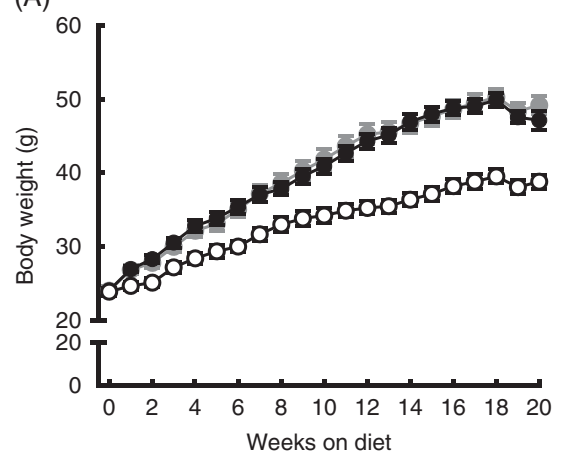

(B)

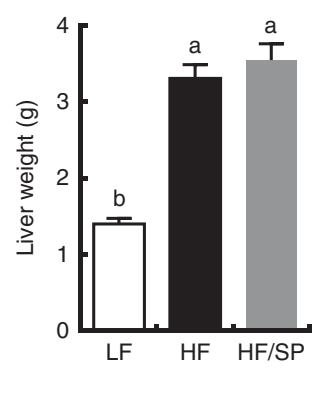

(C)

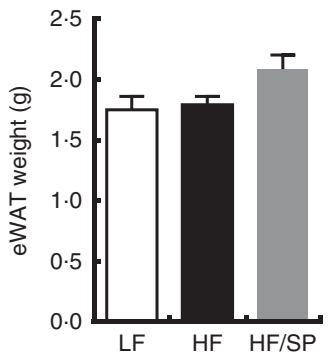

Fig. 1. Effect of Spirulina platensis (SP) supplementation on body and tissue weights in a mouse model of liver fibrosis. Mice fed a low-fat (LF, - - -), a high-fat (HF, - $/$ /high-sucrose/high-cholesterol diet or an HF diet supplemented with $2.5 \%$ (w/w) SP (HF/SP, - - -) for 20 weeks. (A) Body weight of mice throughout the 20 week feeding period. Liver weight (B) and epididymal white adipose tissue (eWAT) weight $(\mathrm{C})$ after 20 weeks on experimental diets. Values are means ( $n 12-15$ per group), with the standard errors of the mean represented by vertical bars. ${ }^{\text {a,b }}$ Mean values with unlike letters were significantly different from each other $(P<0.05)$.

(A)

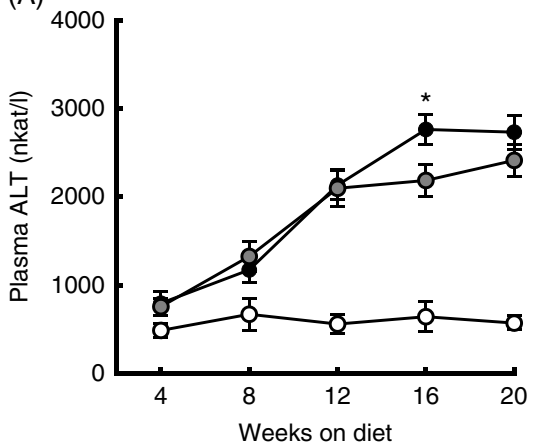

(B)
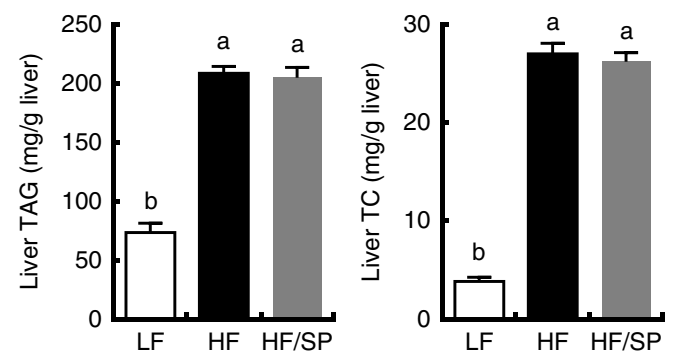

(C)

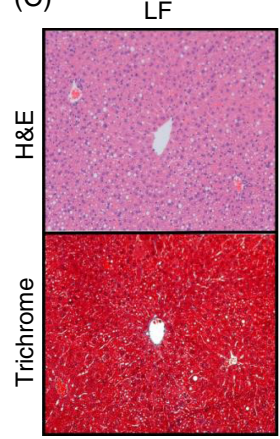

HF

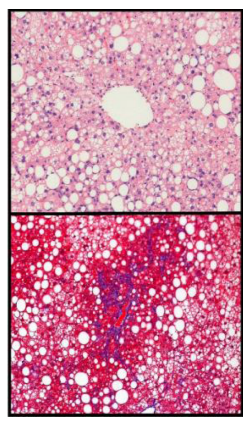

$\mathrm{HF} / \mathrm{SP}$

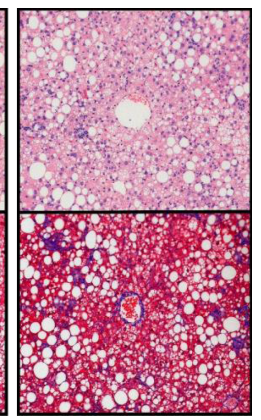

(D)
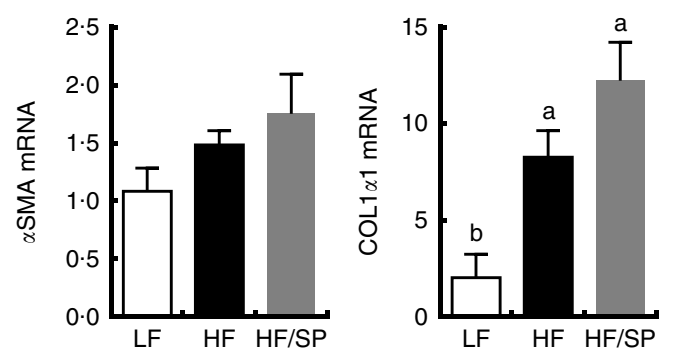

Fig. 2. Effect of Spirulina platensis (SP) supplementation on liver damage, lipid and fibrosis in a mouse model of liver fibrosis. Mice fed a low-fat (LF, $-0-$ ), a high-fat (HF, - - ) /high-sucrose/high-cholesterol diet or an HF diet supplemented with $2.5 \%$ (w/w) SP (HF/SP, - - ) for 20 weeks. (A) Plasma alanine aminotransferase (ALT) levels throughout the 20 weeks' feeding period. (B) Liver TAG (left) and total cholesterol (TC, right) levels. (C) Representative haematoxylin and eosin (H\&E) staining (top) and trichrome staining (bottom) of formalin-fixed liver sections. (D) Hepatic expression of $a$-smooth muscle actin (aSMA) and collagen 1a1 (COL1a1). Values are means $\left(n 12-15\right.$ per group), with standard errors of the mean represented by vertical bars. ${ }^{\text {a,b }}$ Mean values with unlike letters were significantly different from each other $(P<0.05)$. * Mean value was significantly different from that for the HF/SP group $(P<0.05)$.

via portal vein blood flow ${ }^{(11,26)}$. Spleen weight was significantly increased by HF and the HF/SP diets compared with the LF diet (Fig. 5(A)), suggesting that mice on the diets were likely to experience portal hypertension, which is characteristic of liver fibrosis ${ }^{(27,28)}$. Splenocytes isolated from mice on the HF/SP diet had significantly lower expression levels of basal IL- $1 \beta$ compared with those isolated from the HF group (Fig. 5(B)), while a decrease in IL-6 expression by SP was trending towards significance. Also, the splenocytes from mice on the HF or HF/SP diet for 16 weeks were tested ex vivo for their LPS sensitivity. The splenocytes from mice fed the HF/SP diet had significantly lower mRNA levels of LPS-induced IL- $1 \beta$ and TNF- $\alpha$ as well as TNF- $\alpha$ secretion compared with those from the HF group (Fig. 5(C) and 5(D)).

\section{Discussion}

NAFLD is a major global health problem with few available treatments $^{(1)}$. Therefore, prevention of NAFLD is important to 
(A)

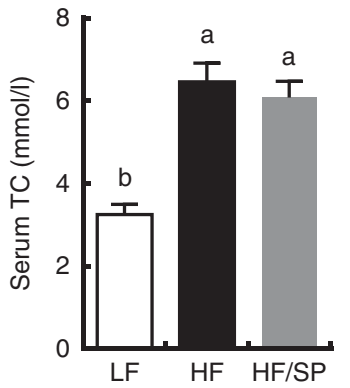

(B)

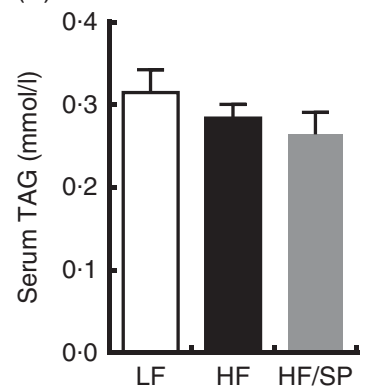

(C)

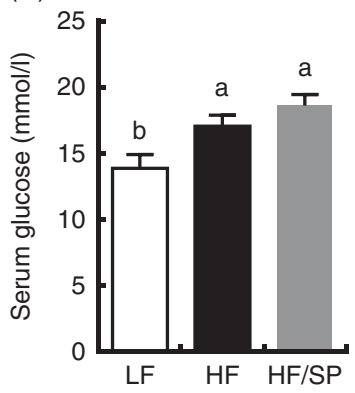

(D)

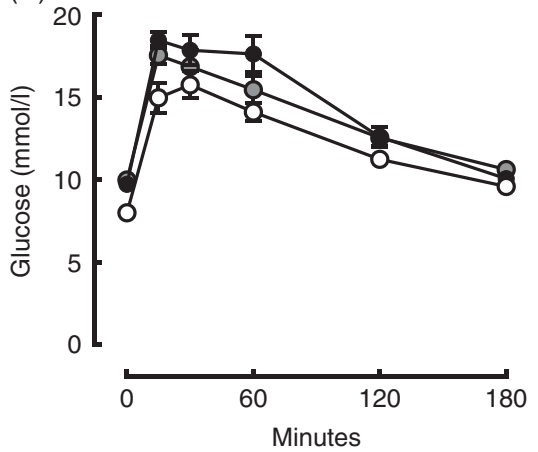

(E)

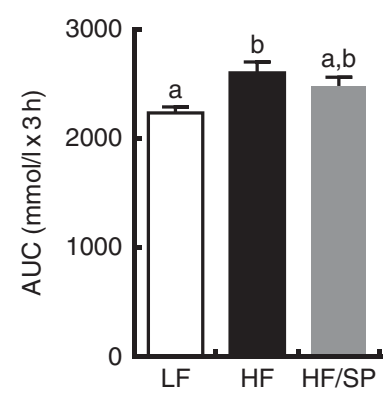

Fig. 3. Effect of Spirulina platensis (SP) supplementation on serum lipids and glucose tolerance in a mouse model of liver fibrosis. Mice fed a low-fat (LF, $-0-$ ), a high-fat (HF, - - - /high-sucrose/high-cholesterol diet or an HF diet supplemented with $2.5 \%$ (w/w) SP (HF/SP, —- - ) for 20 weeks. Serum levels of total cholesterol (TC) (A), TAG (B) and glucose (C). (D) Oral glucose tolerance test was conducted 18 weeks after mice were on the experimental diets. (E) The AUC analysis of oral glucose tolerance test. Values are means ( $n$ 12-15 per group), with standard errors of the mean represented by vertical bars. ${ }^{a, b}$ Mean values with unlike letters were significantly different from each other $(P<0.05)$.

minimise global health burden. In the present study, we used an obesogenic diet high in fat, sucrose and cholesterol to induce liver fibrosis for the investigation of the role of SP in the prevention of diet-induced liver fibrosis. We found that SP supplementation had a minimal effect on blood and tissue lipids, whole body energy metabolism and liver fibrosis in this mouse model. However, supplementation of SP reduced basal and LPS-induced inflammatory gene expression and cytokine secretion in splenocytes, supporting the anti-inflammatory effect of SP in vivo. Although there were no appreciable effects of SP in this mouse model, SP may be beneficial for conditions where splenocytes are the source of inflammation such as myocardial infarction ${ }^{(29)}$, ischaemic brain injury ${ }^{(30)}$ and ischaemia-reperfusion injury ${ }^{(31)}$. Furthermore, conditions that involve chronic inflammation such as colitis and arthritis may also benefit from SP supplementation.

In the current study, SP supplementation did not alter blood or liver lipid levels. This is consistent with the findings from our previous studies with SP in several mouse models ${ }^{(23,32,33)}$. Supplementation of $2.5 \%(\mathrm{w} / \mathrm{w})$ or $5.0 \%(\mathrm{w} / \mathrm{w})$ SP to an AIN-93G/M diet for 6 months in $\mathrm{C} 57 \mathrm{BL} / 6 \mathrm{~J}$ mice did not alter plasma lipids or liver lipids ${ }^{(32)}$. In apoE knockout mice, supplementation of $5.0 \%$ (w/w) SP in an HF/high-cholesterol diet for 12 weeks did not alter plasma or liver lipids, while it reduced atherosclerosis development ${ }^{(23)}$. In another study, when $\mathrm{C} 57 \mathrm{BL} / 6 \mathrm{~J}$ mice were fed an $\mathrm{HF} /$ high-sucrose diet supplemented with or without $0.25 \%$ SPE for 16 weeks, there were no changes in plasma lipids by $\mathrm{SPE}^{(33)}$. However, we observed a significant reduction in inflammatory gene expression in splenocytes and peritoneal macrophages from mice fed SPE. Collectively, our studies including the present study consistently indicate that SP has a minimal effect on hyperlipidaemia, but it exerts a potent anti-inflammatory function.

The 'two-hit model' for NAFLD development and progression includes the accumulation of fat in the liver, that is, the first hit, followed by the second hit, which includes oxidative stress, lipid peroxidation, pro-inflammatory cytokines, adipokines and mitochondrial dysfunction ${ }^{(4)}$. Although in the current study, we did not observe a reduction in the first hit, that is, lipid accumulation, we found that the splenocytes from mice fed SP had significantly lower basal and LPS-induced inflammatory gene expression. This finding is of significance as the spleen has been demonstrated to be a reservoir for monocytes that rapidly mobilise to sites of tissue injury ${ }^{(34)}$, including the liver ${ }^{(15)}$. Given the association of the spleen and the liver via the portal vein system, such that splenic secretions pass through the liver and to the heart and then on to the general circulation ${ }^{(11)}$, the spleen may play a significant role in the development of liver fibrosis. Indeed, it has been suggested that splenic red pulp macrophages are a major source of TGF- $\beta 1$ and that splenectomy decreased serum TGF- $\beta$ levels significantly while reducing liver fibrosis ${ }^{(26)}$. Furthermore, it has been demonstrated that the spleen supports the infiltration of monocytes into the liver through the secretion of $\mathrm{C}-\mathrm{C}$ motif chemokine ligand 2 and that splenectomy decreased the infiltration of monocytes to reduce liver fibrosis ${ }^{(35)}$. Therefore, the spleen likely contributes to the development of liver fibrosis via monocyte infiltration and cytokine secretion and that SP may attenuate this liver inflammation through affecting the spleen. 
(A)

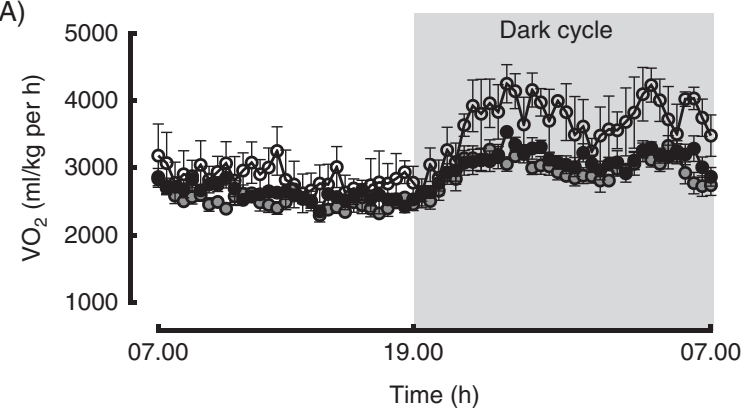

(B)

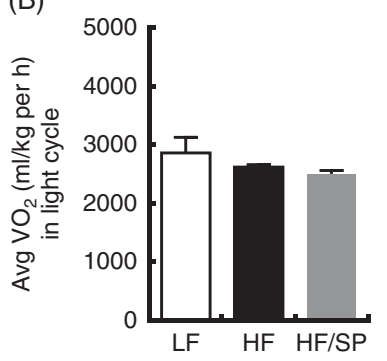

(D)

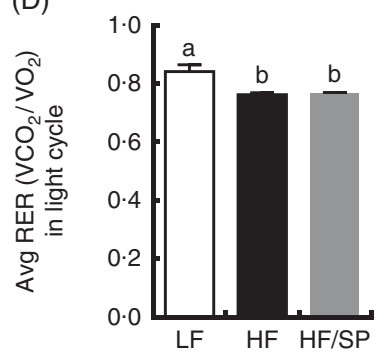

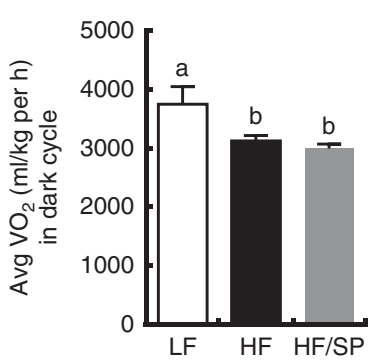
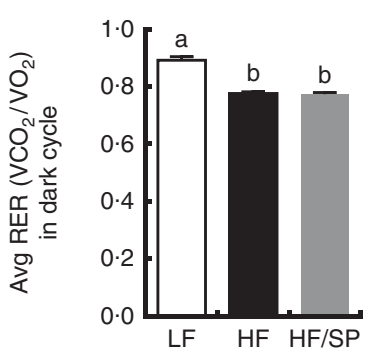
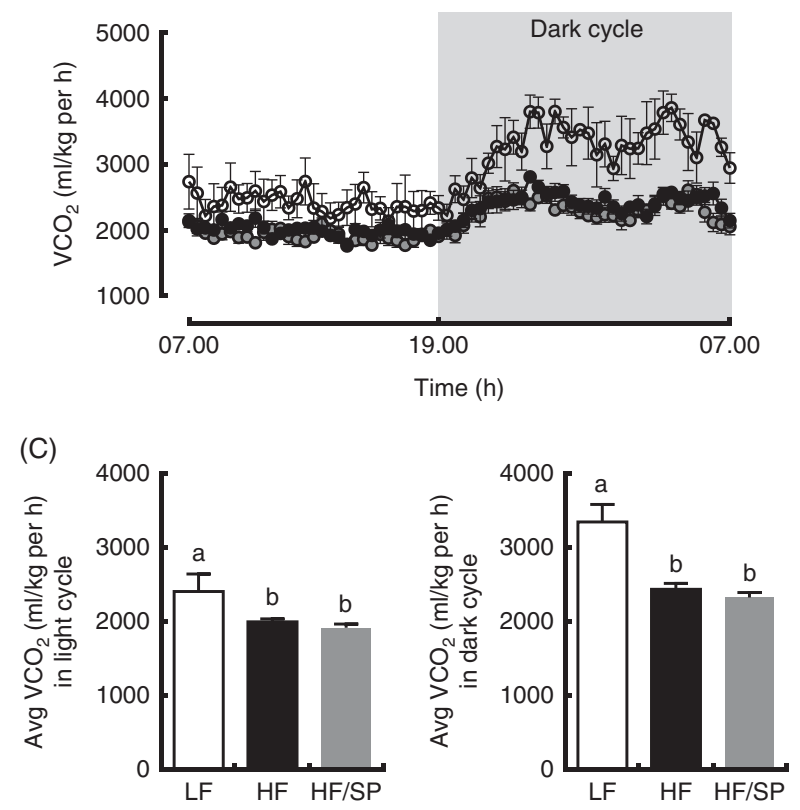

(E)
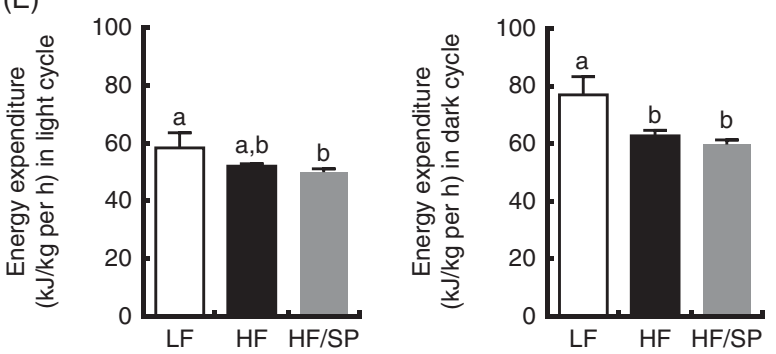

Fig. 4. Metabolic rates of mice fed Spirulina platensis (SP). Mice fed a low-fat (LF, $-\mathrm{O}-)$, a high-fat (HF, $-\mathbf{O}-$ )/high-sucrose/high-cholesterol diet or an $\mathrm{HF}$ diet

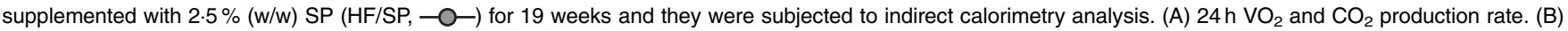
Average $\mathrm{VO}_{2}$ rate in light and dark cycle. (C) Average $\mathrm{CO}_{2}$ production rate in light and dark cycle. (D) Average respiratory exchange ratio (RER) of mice in light and dark cycle. (E) Energy expenditure in light and dark cycle. Values are means ( $n 4$ for LF, 8 for HF and HF/SP), with standard errors of the mean represented by vertical bars. ${ }^{a, b}$ Mean values with unlike letters were significantly different from each other $(P<0.05)$.

The notable effect of SP supplementation observed in the present study is that SP reduced the basal and LPS-induced expression of inflammatory genes in splenocytes. This observation is consistent with the findings in our previous studies that demonstrated the anti-inflammatory effect of SP supplementation or its extract in the spleen ${ }^{(22,33)}$. As mentioned previously, in apoE knockout mice, we found that SP supplementation reduced the secretion of IL- 6 from splenocytes and the formation of aortic lesions without altering plasma lipids ${ }^{(22,23)}$. Furthermore, SP supplementation significantly lowered the cluster of differentiation 68 (CD68)-stained area in the aortic root, suggesting reduced monocyte infiltration ${ }^{(22)}$. Considering the role of the spleen as a reservoir of monocytes that deploys to the site of injury $^{(34)}$, it is possible that SP may reduce the infiltration of monocytes to damaged tissues. It is noteworthy that we observed that SP supplementation has reduced inflammatory gene expression and cytokine secretion across several mouse models and experimental diets. This suggests that SP possesses a robust and spleen-specific effect that warrants further investigation.

Reduction of inflammatory gene expression by SP may be due to changes in the population of immune cells found in the spleen, which has been demonstrated to change in response to obesity $^{(36,37)}$. Alternatively, components found in SP may also reduce inflammatory gene expression in splenocytes by inhibiting the Toll-like receptor 4 signalling pathways. Indeed, SP has been demonstrated to contain high levels of $\gamma$-linolenic $\operatorname{acid}^{(38)}$, which can reduce LPS-induced inflammatory gene expression in monocytes and macrophages ${ }^{(39,40)}$. In addition, SP has high levels of phycocyanin and carotenoids that can exert anti-inflammatory effects ${ }^{(41-43)}$. It is possible that the antiinflammatory effect of SP in the spleen is due to one of these bioactive components but could also be the net result of some or all of them. Work in our laboratory is underway to identify the anti-inflammatory bioactives in SP.

Splenic macrophages have been proposed to be a major contributor of cytokines in the development of liver fibrosis ${ }^{(26)}$. Despite reductions in inflammatory gene expression in splenocytes from mice fed the HF/SP diet for 16 weeks, the lack of reductions in the expression of fibrogenic genes in the liver by the diet after 20 weeks suggests that the anti-inflammatory effect of SP could not overcome the deleterious effect of the HF diet. In the current diet-induced model of liver fibrosis, as there are multiple 
(A)

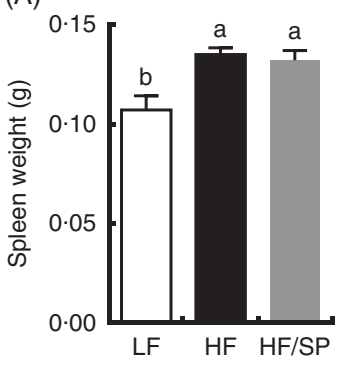

(C)

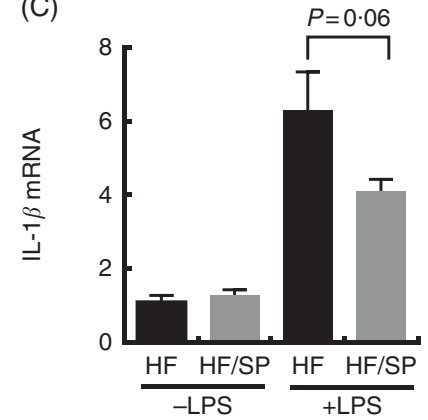

(B)
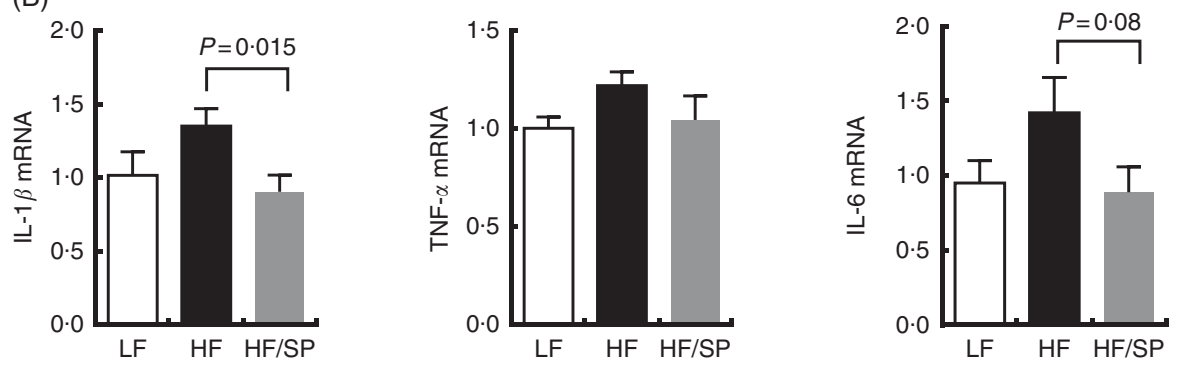

(D)
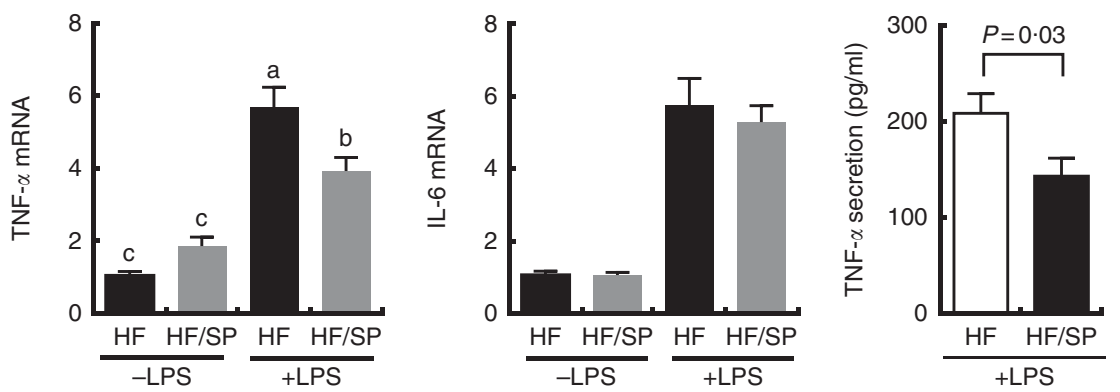

Fig. 5. Anti-inflammatory effects of SP in splenocytes from a mouse model of liver fibrosis. Mice fed a low-fat (LF), a high-fat (HF)/high-sucrose/high-cholesterol diet or an HF diet supplemented with $2.5 \%(\mathrm{w} / \mathrm{w}) \mathrm{SP}(\mathrm{HF} / \mathrm{SP})$ for 16 or 20 weeks. Spleen weight (A) and mRNA expression of pro-inflammatory cytokines in the splenocytes (B) of mice on experimental diets for 20 weeks. $n$ 8-9 per group. Splenocytes were isolated from mice on the experimental diets for 16 weeks, and subsequently, they were stimulated with $500 \mathrm{ng} / \mathrm{ml}$ of lipopolysaccharide (LPS) for $20 \mathrm{~h}$ to measure the expression of pro-inflammatory cytokines (C) and TNF- $a$ secretion (D). $n$ 8-10 per group. Values are means, with standard errors of the mean represented by vertical bars. ${ }^{a, b, c}$ Mean values with unlike letters were significantly different from each other $(P<0.05)$.

stressors for liver damage, it is possible that a mere reduction in splenocyte inflammatory gene expression may not be sufficient to overcome other sources of inflammation such as Kupffer cells and bone marrow-derived macrophages. At 16 weeks, mice on the HF/SP diet had significantly lower plasma ALT compared with the HF control, which was not observed at 20 weeks. This suggests that SP supplementation at $2.5 \%(\mathrm{w} / \mathrm{w})$, which is equivalent to about $10 \mathrm{~g}$ of SP a day for humans, exerted hepatoprotective effects but only up to a certain point and that a higher supplemental level of SP may be effective in reducing liver fibrosis.

In conclusion, we demonstrated that SP at the supplementation level of $2.5 \%(\mathrm{w} / \mathrm{w})$ did not prevent the development of liver fibrosis induced by a diet high in fat, sucrose and cholesterol in mice. However, SP supplementation reduced basal and LPS-induced inflammatory gene expression and secretion in splenocytes. Our data suggest that SP exerts antiinflammatory effects, especially in the spleen. Whether a higher supplementation level of SP may prevent the development of liver fibrosis is warranted. Also, further studies are needed to evaluate the role of SP supplementation in mitigating other inflammatory diseases or conditions where spleen is the primary source of inflammation.

\section{Acknowledgements}

This study was supported by USDA NIFA (2017-67012-26096) to T. X. P. and USDA Multistate Hatch CONS 00916 to J.-Y. L.

T. X. P., Y. L, M. B., S. H., H. K., M.-B. K. and Y.-K. P. carried out experiments and performed data analysis; T. X. P., Y.-K. P. and J.-Y. L. wrote the manuscript and J.-Y. L. designed and led the study.

The authors declare that there are no conflicts of interest.

\section{References}

1. Younossi Z, Anstee QM, Marietti M, et al. (2018) Global burden of NAFLD and NASH: trends, predictions, risk factors and prevention. Nat Rev Gastroenterol Hepatol 15, 11-20.

2. Younossi ZM, Blissett D, Blissett R, et al. (2016) The economic and clinical burden of nonalcoholic fatty liver disease in the United States and Europe. Hepatology 64, 1577-1586.

3. Hardy T, Oakley F, Anstee QM, et al. (2016) Nonalcoholic fatty liver disease: pathogenesis and disease spectrum. Annu Rev Pathol 11, 451-496.

4. Paschos P \& Paletas K (2009) Non alcoholic fatty liver disease and metabolic syndrome. Hippokratia 13, 9-19.

5. Marchesini G, Brizi M, Morselli-Labate AM, et al. (1999) Association of nonalcoholic fatty liver disease with insulin resistance. Am J Med 107, 450-455.

6. Loomba R, Abraham M, Unalp A, et al. (2012) Association between diabetes, family history of diabetes, and risk of nonalcoholic steatohepatitis and fibrosis. Hepatology 56, 943-951.

7. Friedman SL (1999) Stellate cell activation in alcoholic fibrosis - an overview. Alcohol Clin Exp Res 23, 904-910.

8. Parola M \& Robino G (2001) Oxidative stress-related molecules and liver fibrosis. J Hepatol 35, 297-306.

9. Pellicoro A, Ramachandran P, Iredale JP, et al. (2014) Liver fibrosis and repair: immune regulation of wound healing in a solid organ. Nat Rev Immunol 14, 181-194.

10. Koyama Y \& Brenner DA (2017) Liver inflammation and fibrosis. J Clin Invest 127, 55-64. 
11. Li L, Duan M, Chen W, et al. (2017) The spleen in liver cirrhosis: revisiting an old enemy with novel targets. J Transl Med 15, 111-121.

12. Liu P, Li P, He W, et al. (2009) Liver and spleen volume variations in patients with hepatic fibrosis. World J Gastroenterol 15, 3298-3302.

13. Tarao K, Hoshino H, Motohashi I, et al. (1989) Changes in liver and spleen volume in alcoholic liver fibrosis of man. Hepatology 9, 589-593.

14. Jeong WK (2017) Hepatic and splenic volumetry could be used as an imaging parameter to evaluate fibrosis grades of the diffuse liver disease including nonalcoholic fatty liver disease. Gut Liver 11, 577-578.

15. Mandal M, Gardner CR, Sun R, et al. (2016) The spleen as an extramedullary source of inflammatory cells responding to acetaminophen-induced liver injury. Toxicol Appl Pharmacol 304, 110-120.

16. Yada A, Iimuro Y, Uyama N, et al. (2015) Splenectomy attenuates murine liver fibrosis with hypersplenism stimulating hepatic accumulation of Ly-6C(lo) macrophages. J Hepatol 63, 905-916.

17. Younossi ZM (2008) Review article: current management of non-alcoholic fatty liver disease and non-alcoholic steatohepatitis. Aliment Pharmacol Ther 28, 2-12.

18. Samuels R, Mani UV, Iyer UM, et al. (2002) Hypocholesterolemic effect of spirulina in patients with hyperlipidemic nephrotic syndrome. J Med Food 5, 91-96.

19. Mao TK, Van de Water J \& Gershwin ME (2005) Effects of a spirulina-based dietary supplement on cytokine production from allergic rhinitis patients. J Med Food 8, 27-30.

20. Coskun ZK, Kerem M, Gurbuz N, et al. (2011) The study of biochemical and histopathological effects of spirulina in rats with TNBS-induced colitis. Bratisl Lek Listy 112, 235-243.

21. Rasool M, Sabina EP \& Lavanya B (2006) Anti-inflammatory effect of Spirulina fusiformis on adjuvant-induced arthritis in mice. Biol Pharm Bull 29, 2483-2487.

22. Ku CS, Pham TX, Park Y, et al. (2013) Edible blue-green algae reduce the production of pro-inflammatory cytokines by inhibiting NF-kappaB pathway in macrophages and splenocytes. Biochim Biophys Acta 1830, 2981-2988.

23. Ku CS, Kim B, Pham TX, et al. (2015) Blue-green algae inhibit the development of atherosclerotic lesions in apolipoprotein $\mathrm{E}$ knockout mice. J Med Food 18, 1299-1306.

24. Park YK, Rasmussen HE, Ehler SJ, et al. (2008) Repression of proinflammatory gene expression by lipid extract of Nostoc commune var sphaeroides Kutzing, a blue-green alga, via inhibition of nuclear factor-kappa B in RAW 264.7 macrophages. Nutr Res 28, 83-91.

25. Rasmussen HE, Blobaum KR, Park YK, et al. (2008) Lipid extract of Nostoc commune var. sphaeroides Kutzing, a bluegreen alga, inhibits the activation of sterol regulatory element binding proteins in HepG2 cells. J Nutr 138, 476-481.

26. Akahoshi T, Hashizume M, Tanoue K, et al. (2002) Role of the spleen in liver fibrosis in rats may be mediated by transforming growth factor beta-1. J Gastroenterol Hepatol 17, 59-65.

27. Rockey DC (2006) Hepatic fibrosis, stellate cells, and portal hypertension. Clin Liver Dis 10, 459-479, vii-viii.
28. Bolognesi M, Merkel C, Sacerdoti D, et al. (2002) Role of spleen enlargement in cirrhosis with portal hypertension. Dig Liver Dis 34, 144-150.

29. Leuschner F, Rauch PJ, Ueno T, et al. (2012) Rapid monocyte kinetics in acute myocardial infarction are sustained by extramedullary monocytopoiesis. J Exp Med 209, 123-137.

30. Kim E, Yang J, Beltran CD, et al. (2014) Role of spleen-derived monocytes/macrophages in acute ischemic brain injury. J Cereb Blood Flow Metab 34, 1411-1419.

31. Hsiao HM, Fernandez R, Tanaka S, et al. (2018) Spleenderived classical monocytes mediate lung ischemia-reperfusion injury through IL-1beta. J Clin Invest 128, 2833-2847.

32. Yang Y, Park Y, Cassada DA, et al. (2011) In vitro and in vivo safety assessment of edible blue-green algae, Nostoc commune var. sphaeroides Kutzing and Spirulina platensis. Food Chem Toxicol 49, 1560-1564.

33. Pham TX, Park YK, Bae M, et al. (2017) The potential role of an endotoxin tolerance-like mechanism for the antiinflammatory effect of Spirulina platensis organic extract in macrophages. J Med Food 20, 201-210.

34. Swirski FK, Nahrendorf M, Etzrodt M, et al. (2009) Identification of splenic reservoir monocytes and their deployment to inflammatory sites. Science 325, 612-616.

35. Li L, Wei W, Li Z, et al. (2018) The spleen promotes the secretion of CCL2 and supports an M1 dominant phenotype in hepatic macrophages during liver fibrosis. Cell Physiol Biochem 51, 557-574.

36. Zou J, Lai B, Zheng $\mathrm{M}$, et al. (2017) $\mathrm{CD}^{4+} \mathrm{T}$ cells memorize obesity and promote weight regain. Cell Mol Immunol 15, 630-639.

37. Boi SK, Buchta CM, Pearson NA, et al. (2016) Obesity alters immune and metabolic profiles: new insight from obeseresistant mice on high-fat diet. Obesity (Silver Spring) 24, 2140-2149.

38. Ronda SR \& Lele SS (2008) Culture conditions stimulating high gamma-linolenic acid accumulation by Spirulina platensis. Braz J Microbiol 39, 693-697.

39. Chang CS, Sun HL, Lii CK, et al. (2010) Gamma-linolenic acid inhibits inflammatory responses by regulating NF-kappaB and AP-1 activation in lipopolysaccharide-induced RAW 264.7 macrophages. Inflammation 33, 46-57.

40. Furse RK, Rossetti RG, Seiler CM, et al. (2002) Oral administration of gammalinolenic acid, an unsaturated fatty acid with anti-inflammatory properties, modulates interleukin-1beta production by human monocytes. J Clin Immunol 22, 83-91.

41. Gonzalez R, Rodriguez S, Romay C, et al. (1999) Antiinflammatory activity of phycocyanin extract in acetic acidinduced colitis in rats. Pharmacol Res 39, 55-59.

42. Cherng SC, Cheng SN, Tarn A, et al. (2007) Anti-inflammatory activity of $c$-phycocyanin in lipopolysaccharide-stimulated RAW 264.7 macrophages. Life Sci 81, 1431-1435.

43. Ciccone MM, Cortese F, Gesualdo M, et al. (2013) Dietary intake of carotenoids and their antioxidant and antiinflammatory effects in cardiovascular care. Mediators Inflamm 2013, 782137. 